\title{
Artificial Insemination: Current and Future Trends
}

\author{
Jane M. Morrell \\ Swedish University of Agricultural Sciences, Uppsala, \\ Sweden
}

\section{Introduction}

The chapter will deal with the use of artificial insemination (AI) in animals and humans, both currently and in the future, with particular emphasis on comparative aspects between species. Although AI (in the form of intrauterine insemination) is not frequently used in human patients, it is the most commonly used method of breeding food production animals in developed countries, with more than $90 \%$ pigs and almost the same proportion of dairy cattle bred by this method in the European Union and North America. This chapter will explain the advantages and disadvantages of using AI, the various methodologies used in different species, and how AI can be used to improve reproductive efficiency in farm animals, sport animals, and human patients. To finish, some speculation is made about future trends for this biotechnology.

\subsection{What is artificial insemination (AI)?}

Artificial insemination (AI) is the manual placement of semen in the reproductive tract of the female by a method other than natural mating. It is one of a group of technologies commonly known as "assisted reproduction technologies" (ART), whereby offspring are generated by facilitating the meeting of gametes (spermatozoa and oocytes). ART may also involve the transfer of the products of conception to a female, for instance if fertilization has taken place in vitro or in another female. Other techniques encompassed by ART include the following: in vitro fertilization (IVF) where fertilization takes place outside the body; intracytoplasmic sperm injection (ICSI) where a single spermatozoon is caught and injected into an oocyte; embryo transfer (ET) where embryos that have been derived either in vivo or in vitro are transferred to a recipient female to establish a pregnancy; gamete intrafallopian transfer (GIFT) where spermatozoa are injected into the oviduct to be close to the site of fertilization in vivo; and cryopreservation, where spermatozoa or embryos, or occasionally oocytes, are cryopreserved in liquid nitrogen for use at a later stage.

AI has been used in the majority of domestic species, including bees, and also in human beings. It is the most commonly used ART in livestock, revolutionising the animal breeding industry during the $20^{\text {th }}$ century. In contrast to medical use, where intra-uterine insemination (IUI) is used only occasionally in human fertility treatment, AI is by far the most common method of breeding intensively kept domestic livestock, such as dairy cattle (approximately 80\% in Europe and North America), pigs (more than 90\% in Europe and North America) and turkeys (almost $100 \%$ in intensive production). AI is increasing in 
horses, beef cattle and sheep, and has been reported in other domestic species such as dogs, goats, deer and buffalo. It has also been used occasionally in conservation breeding of rare or endangered species, for example, primates, elephants and wild felids. The other ARTs in animals are generally confined to specialist applications or for research purposes, since the cost would be prohibitive for normal livestock breeding. In contrast, IUI is used less often in human fertility treatments than IVF or ICSI.

\subsection{Advantages and disadvantages of artificial insemination}

$\mathrm{AI}$ in animals was originally developed to control the spread of disease, by avoiding the transport of animals with potential pathogens to other animal units for mating and by avoiding physical contact between individuals. The use of semen extenders containing antibiotics also helped to prevent the transmission of bacterial diseases. The advantages and disadvantages of $\mathrm{AI}$ are as follows:

\section{Advantages:}

- $\quad$ AI helps prevents the spread of infectious or contagious diseases, that can be passed on when animals are in close contact or share the same environment;

- The rate of genetic development and production gain can be increased, by using semen from males of high genetic merit for superior females;

- It enables breeding between animals in different geographic locations, or at different times (even after the male's death);

- Breeding can occur in the event of physical, physiological or behavioural abnormalities;

- $\mathrm{AI}$ is a powerful tool when linked to other reproductive biotechnologies such as sperm cryopreservation, sperm sexing;

- $\quad$ AI can be used in conservation of rare breeds or endangered species.

Disadvantages:

- Some males shed virus in semen without clinical signs of disease ("shedders").

- Some bacterial pathogens are resistant to the antibiotics in semen extenders or can avoid their effects by forming bio-films;

- There has been a decline in fertility in dairy cattle and horses associated with an increase in AI;

- The focus on certain individuals may result in loss of genetic variation.

\subsubsection{Viruses in semen}

Cryopreserved semen doses can be "quarantined" until the male is shown to have been free of disease at the time of semen collection. In contrast, the short shelf-life of fresh semen doses means that they must be inseminated into the female before the disease-free status of the male has been established. Breeding sires used for semen collection are tested routinely for the presence of antibodies in serum as being indicative of past infection, but some viruses, e.g. equine arteritis virus, may be shed in semen for several weeks before there is evidence of sero-conversion. In other cases, usually of congenital infection, individuals may be permanent virus "shedders" without ever developing antibodies. Semen from these individuals represents a source of pathogens for disease transmission to naive females.

\subsubsection{Bacteria in semen}

Normally, in a healthy male, the ejaculate itself does not contain microorganisms, but contamination occurs at semen collection from the prepuce and foreskin, the male's 
abdomen and the environment. Semen processing from livestock usually takes place without access to a laminar air flow hood, resulting in potential contamination from the laboratory environment. Antibiotics are added to semen extenders to limit the growth of these contaminants and prevent disease in the inseminated female. Although the female reproductive tract has well-developed physiological mechanisms for dealing with contamination introduced during mating, these can be overwhelmed by bacteria multiplying in semen extenders or where semen is deposited in a non-physiological location.

\subsubsection{Antibiotics in semen extenders}

The addition of antibiotics to semen extenders is controlled by government directives, both nationally and internationally, which state the types of antibiotic to be used and also their concentrations. In general, there is a tendency to use broad spectrum, highly potent antibiotics in various combinations to reduce sperm toxicity. However, these antibiotics may exacerbate the development of resistance, both for the people handling the semen extenders and in the environment during the disposal of unused extenders or semen doses. The scale of the problem becomes apparent if one considers that approximately four million liters of boar semen extender containing antibiotics are used in Europe alone per year.

\section{Pre-requisites for $\mathrm{Al}$}

Pre-requisites for AI include a supply of semen, reliable methods for oestrus detection in the female and a means of inserting the semen into the female reproductive tract.

\subsection{Collection of semen}

In most domestic animals, semen is collected by means of an artificial vagina, for example, bull, ram, stallion, after allowing the male to mount either an oestrous female or a phantom. The artificial vagina consists of a lubricated liner inserted into an outer jacket, the space between the two being filled with warm water. The pressure can be increased by adding air. The ejaculate is deposited into an insulated collecting vessel attached to one end of the liner. Boar and dog semen is usually collected by manual stimulation.

In some species that are accustomed to being handled, it is possible to obtain semen by vaginal washing after natural mating, for example, dogs and marmoset monkeys. However, in this case the spermatozoa have already been exposed to vaginal secretions which may be detrimental to sperm survival. Human males can usually supply a sample by masturbation, except in the case of spinal injury when electroejaculation may be necessary. Some other primates can be trained to supply a semen sample on request in the same manner. For other species, for example, most non-domestic species, electroejaculation represents the only possibility for obtaining a semen sample. The problem with electroejaculation is that the secretions of the accessory glands may not be present in the usual proportions, which may have a detrimental effect on sperm survival.

\subsubsection{Constituents of semen}

Semen consists of spermatozoa contained in a watery fluid known as seminal plasma that represents the combined secretions of the different accessory glands, such as the seminal vesicles, bulbourethral gland and prostate. The relative contributions of these different 
glands vary between species. In some species, such a most primates, the semen coagulates immediately after ejaculation and then liquefies over a period of approximately 30 minutes. In most other species, the ejaculate remains liquid, the exception being in camelids where the seminal plasma is highly viscous and does not liquefy readily in vitro. The addition of enzymes has been suggested as a means of liquefying primate or camelid semen. However, all the enzymes tested thus far (collagenase, fibrinolysin, hyaluronidase and trypsin) have been seen to cause acrosomal damage in spermatozoa (Wani et al., 2007) and are contraindicated if the spermatozoa are to be used for AI. Recent advances have shown that camelid semen, extended 1:1 volume to volume, will liquefy in $60-90 \mathrm{~min}$ at $37^{\circ} \mathrm{C}$.

Seminal plasma contains an energy source (often fructose), proteins and various ions such as calcium, magnesium, zinc and bicarbonate. Seminal plasma not only activates the spermatozoa, which have been maintained in a quiescent state in the epididymis, but also functions as a transport medium to convey the spermatozoa into the female reproductive tract and to stimulate the latter to allow spermatozoa to swim to the site of fertilization. It has been suggested that seminal plasma, at least in horses, is also a modulator of sperminduced inflammation, which is thought to play an important role in sperm elimination from the female reproductive tract (Troedsson et al., 2001). Various proteins in the seminal plasma, such as spermadhesins and the so-called CRISP proteins (CRISP $=$ cysteine-rich secretory proteins) are thought to be associated with sperm fertility. It is likely that these proteins bind to spermatozoa immediately, setting in motion a sequence of intracellular events via a second-messenger pathway. In some species, small membrane-bound vesicles have also been identified in seminal plasma, apparently originating from different accessory glands in various species. These vesicles, variously named prostasomes, vesiculosomes, or epididysomes depending on their origin, fuse with the sperm outer membrane, increasing motility and possibly being involved in sperm capacitation and acquisition of fertilizing ability. However, their exact mechanism of action has yet to be elucidated.

Seminal factors promote sperm survival in the female reproductive tract, modulate the female immune response tolerate the conceptus, and to condition the uterine environment for embryo development and the endometrium for implantation (Robertson, 2005). The mechanism of action in the endometrium is via the recruitment and activation of macrophages and granulocytes, and also dendritic re-modelling, that improve endometrial receptivity to the implanting embryo. Cytokine release has embryotrophic properties and may also influence tissues outside the reproductive tract.

Exposure to semen induces cytokine activation into the uterine luminal fluid and epithelial glycocalyx lining the luminal space. These cytokines interact with the developing embryo as it traverses the oviduct and uterus prior to implantation. Several cytokines are thought to be involved, for example granulocyte-macrophage colony stimulating factor (GM-CSF), a principle cytokine in the post-mating inflammatory response, targets the pre-implantation embryo to promote blastocyst formation, increasing the number of viable blastomeres by inhibiting apoptosis and facilitating glucose uptake (Robertson et al., 2001). Interleukin-6 (IL-6) and leukocyte inhibitory factor (LIF) are similarly induced after exposure to semen (Gutsche et al., 2003; Robertson et al., 1992).

Clinical studies in humans showed acute and cumulative benefits of exposure to seminal fluid but also a partner-specific route of action. Live birth rates in couples undergoing fertility treatments are improved if women engage in intercourse close to embryo transfer (Bellinge et al., 1986; Tremellen et al., 2000). The use of seminal plasma pessaries by women suffering from recurrent spontaneous abortion is reported to improve pregnancy success 
(Coulam and Stern, 1993, cited in Robertson, 2005). Partner-specificity of the response is suggested by increased rates of preeclampsia in pregnancies from donor oocytes or semen when prior exposure to the donor sperm or conceptus antigens has not occurred (Salha et al., 1999).

\subsubsection{Semen processing}

Although seminal plasma plays such an important role in activating spermatozoa and in the female reproductive tract, it is detrimental to long-term sperm survival outside the body. Under physiological conditions, spermatozoa are activated by seminal plasma at ejaculation and then swim away from the site of semen deposition in the female. It is only during in vitro storage that spermatozoa become exposed to seminal plasma long-term. Thus it is customary to add a semen extender to the semen, to dilute toxic elements in seminal plasma, to provide nutrients for the spermatozoa during in vitro storage and to buffer their metabolic by-products. The addition of extender also permits the semen to be divided into several semen doses, each containing a specific number of spermatozoa that has been determined to be optimal for good fertility in inseminated females.

\subsubsection{Semen preservation}

Semen is used either immediately after collection ("fresh") for example turkeys, human beings; after storage at a reduced temperature ("stored") for example horses, pigs, dogs; or after freezing and thawing ("cryopreservation") for example, bulls.

\subsubsection{Fresh semen}

In contrast to animal species, human semen is not extended prior to processing (see previous section) and is not usually kept for more than a few hours before use. Poultry semen cannot be extended as much as is customary for other species since the spermatozoa are adversely affected by increased dilution. Goat semen cannot be kept at $37^{\circ} \mathrm{C}$ because an enzymatic component of the bulbo-urethral gland secretion hydrolyses milk triglycerides into free fatty acids, which adversely affects the motility and membrane integrity of buck spermatozoa (Pellicer-Rubio and Combarnous, 1998). For liquid preservation, goat semen can be stored at $4^{\circ} \mathrm{C}$ although fertility is retained for only $12-24 \mathrm{~h}$. The rate of extension used for stallion semen varies between countries but rates of 1:2, 1:3 or even 1:4 (v/v) semen:extender are common. The standard practice in some countries is to have 500 million or one billion progressively motile stallion spermatozoa for fresh or cooled semen doses respectively. Boar semen doses contain three billion progressively motile spermatozoa.

\subsubsection{Stored semen}

Storing extended semen at reduced temperature helps to extend sperm life by slowing their metabolism as well as by inhibiting bacterial growth. Bacteria grow by utilizing the nutrients in semen extenders, thus competing with spermatozoa for these limited resources, and release metabolic byproducts, thus creating an environment that is not conducive to maintaining viable spermatozoa. Furthermore, as bacteria die, they may release endotoxins that are toxic to spermatozoa. However, cooled stored semen is the method of choice for breeding horses and pigs, enabling the semen dose to be transported to different locations for insemination. Stallion semen is stored at approximately $6^{\circ} \mathrm{C}$ while boar semen is stored between 16 and $18^{\circ} \mathrm{C}$.

Most boar semen doses are sold as cooled doses. In contrast, some stallions produce spermatozoa that do not tolerate cooling, rapidly losing progressive motility. In such cases, 
the only option currently is to use fresh semen doses for AI immediately after semen collection, although a new method of processing, centrifugation through a single layer of colloid, has been shown to solve the problem, as discussed later.

\subsubsection{Cryopreservation}

Semen is most useful for AI if it can be cryopreserved, since this method of preservation ideally enables the semen to be stored for an unlimited period without loss of quality until needed for AI. Since the frozen semen does not deteriorate, it can be quarantined until the male has been shown to be free from disease at the time of semen collection. However, the spermatozoa of various species differ in their ability to withstand cryopreservation: ruminant spermatozoa survive well whereas poultry spermatozoa do not, with less than $2 \%$ retaining their fertilizing ability on thawing (Wishart, 1985). For farm animal breeding, the cost of cryopreservation and the likelihood of a successful outcome following AI must be considered when deciding whether to use fresh, cooled or frozen sperm doses.

The spermatozoa are mixed with a protective solution containing lipoproteins, sugars and a cryoprotectant such as glycerol. These constituents help to preserve membrane integrity during the processes of cooling and re-warming. However, sperm motility must also be maintained, so that the thawed spermatozoa can reach the oocytes after insemination and fertilize them. In most species, the seminal plasma is removed by centrifugation before mixing with the cryoextender, for example, stallion, boar, goat and human semen. The extended semen is packed in straws and frozen in liquid nitrogen vapour before plunging into liquid nitrogen for long-term storage. There is considerable variation in the success of sperm cryopreservation between different species, despite intensive research into the constituents of cryoextenders and the rates of cooling and re-warming. Human spermatozoa can be frozen relatively successfully using commercially available cryoextenders and programmable freezing machines.

\subsection{Oestrus detection and ovulation}

Successful AI also depends on depositing the semen in the female tract at around the time of ovulation. Like human beings, some domestic animals breed throughout the year, for example cattle and pigs, but others show a defined period of reproductive activity known as the breeding season, for example sheep and horses. The onset of the breeding season is controlled by photoperiod. Both of these patterns of reproductive behaviour are characterised by waves of ovarian activity, culminating in ovulation. However, in some other species ovulation occurs in response to the stimulus of mating, for example, cats, rabbits and camels. In spontaneously ovulating species, ovulation occurs at some time during, or shortly after, oestrus, which is the period of time when the female is receptive to the male. Since a successful outcome for AI depends on the deposition of spermatozoa at a suitable time relative to ovulation, oestrus detection is crucial if the female is to be inseminated at the correct time. Males of the same species are, of course, very good at detecting oestrus females, but since many livestock breeding units that practice AI do not have male animals in the vicinity, it is essential that husbandry personnel become good at recognising oestrous behaviour.

Although some domestic animals may show well-developed oestrous behaviour, e.g. dairy cows, others may not. Behavioural signs of oestrus in cows include restlessness or increased activity, vocalization, chin resting, swelling of the vulva, vaginal discharge and mounting other cows, although there are breed differences in the frequency and intensity of these 
signs. In sheep and goats, vulval swelling and vaginal discharge may be seen, and there is usually pronounced male-seeking behaviour. When AI is to be used in sheep, it is usual to synchronize oestrus with hormones: intravaginal sponges impregnated with progestagens are inserted to suppress the ewe's natural ovarian cycle for 12 days. On sponge removal, pregnant mare serum gonadotrophin is administered, with AI taking place at a set time thereafter. Alternatively, a vasectomised ram wearing a marker can be run with the females. When the females are in oestrus, the vasectomised ram marks them as he mounts, thus enabling them to be identified for AI. Oestrous sows and mares can be identified by the behaviour exhibited towards teaser males.

\subsubsection{Induced ovulation}

When AI is performed in species that are normally induced ovulators, such as rabbits, cats and camels, it is necessary to stimulate ovulation. The easiest way to achieve this stimulation is to mate the female with a vasectomised male, but this practice is not desirable from the point of view of disease control and necessitates having vasectomized males available. The most acceptable alternative is to administer luteinising hormone, usually in the form of human chorionic gonadotrophin. However, the major disadvantage is that repeated injections of this foreign protein may cause the female to develop antibodies, thus inactivating subsequent doses.

\subsubsection{Artificially induced ovulation}

Hormones may be administered to spontaneous ovulators to ensure that ovulation occurs at the correct time relative to AI. However, since 2006, the use of hormones in food-producing animals has been forbidden in the European Union, and local regulations may also apply in other parts of the world. Previously most dairy goats in France were inseminated out of the breeding season with deep frozen semen, after induction of oestrus and ovulation by hormonal treatments. This protocol provided a kidding rate of approximately $65 \%$ (Leboeuf et al., 2008). As an alternative to administering artificial hormones, out-of season breeding may be induced by altering the photoperiod or by introducing a buck to the herd. This practice is also widespread in intensive sheep flocks.

\subsection{Deposition of semen in the female}

There are differences between species in the site of semen deposition during natural mating. In ruminants and primates, semen is deposited in the vagina whereas in pigs, dogs, camels and horses, semen deposition is intrauterine. In most species, it is possible to pass an insemination catheter through the cervix, thus enabling semen to be deposited in the uterus during AI. Exceptions are sheep and goats, where the tightly folded nature of the cervix does not permit easy passage of an insemination catheter. The advantages of depositing the semen in the uterus are that the spermatozoa have less far to travel to reach the oviducts and fewer spermatozoa are lost through back-flow. A smaller volume of semen can be used per insemination dose than for intravaginal deposition, thus permitting an ejaculate to be divided into several AI doses, and the cervix, which can act as a barrier to the passage of spermatozoa, is bypassed. A disadvantage, particularly for human IUI, is that seminal plasma is also introduced into the uterus, unless specific steps are taken to separate the spermatozoa from seminal plasma before IUI. 


\section{Species differences in the use of Al}

Despite the fact that the basic principles of AI are the same in all species, there is wide variation in the uptake of this biotechnology in different species.

\subsection{Al in cattle}

In cattle, frozen semen doses are used most widely in Europe and North America, since there are well-established protocols for cryopreserving bull semen. Semen doses typically contain approximately 15 million motile spermatozoa. In New Zealand, however, fresh semen doses are used instead, with AI occurring within $24 \mathrm{~h}$ of semen collection.

\subsection{Al in pigs}

The porcine AI industry uses liquid semen that has been stored for one to several days at 16$18^{\circ} \mathrm{C}$. In contrast, AI with cryopreserved boar spermatozoa results in lower farrowing rates and litter sizes than with cooled, stored spermatozoa, making the use of frozen-thawed sperm doses unattractive for commercial pig breeders. Exceptions to this rule are when semen is transported over long distances, which creates problems in temperature regulation, and in instances where it is vital that the boars can be shown to be free of disease at the time of semen collection. The ability of boar spermatozoa to survive cool storage so well is attributed to low levels of reactive oxygen species (ROS) in semen or to the efficient scavenging of ROS by anti-oxidative components in seminal plasma.

\subsection{Al in horses}

AI has increased in horses in the last 25 years. Initially, fresh semen was used for AI shortly after semen collection, but nowadays the use of cooled semen has largely replaced fresh semen in Europe and North America. The extended semen is cooled to approximately $5^{\circ}$, and transported in insulated containers, together with a cold pack. The fertility of the cooled semen is maintained for approximately $24 \mathrm{~h}$. Frozen semen doses are used infrequently, although this trend may change with the development of better freezing protocols. However, with the increased use of cooled semen, a concomitant decrease in foaling rate has been observed in several countries, such as Finland and Sweden, although the reason for this apparent decline in fertility is unknown. Unlike bulls and boars, which are selected for their semen quality as well as for their potential "genetic merit" in production characteristics (body composition, weight gain, milk production etc), the choice of stallions as breeding sires is based solely on their performance in competition. Thus, considerable variation in semen quality exists between stallions. This variation, coupled with increased use of a wider range of stallions, may be contributing to the observed decline in foaling rate. Other important considerations are the lack of established standard methods for cooling and freezing of stallion spermatozoa, for the sperm concentration in the insemination dose, or for quality control of raw or frozen/thawed spermatozoa.

\section{4 $\mathrm{Al}$ in sheep}

Ram semen differs from stallion and boar semen in consisting of a small volume (a few $\mathrm{mL}$ ) of seminal plasma containing a very high concentration of spermatozoa. In Europe, reproductive research in livestock has tended to focus on cattle and pigs rather than on small ruminants, with the result that sperm handling and cryopreservation for AI is less 
advanced in the latter species. In addition, the anatomy of the female reproductive tract in these species presents more of a barrier to successful insemination than in cattle, since the cervix is tightly folded, making insertion of the insemination catheter difficult. Productivity in sheep and goats could be increased, by improving the quality of the spermatozoa assigned for use in AI, and improving the AI techniques in these species. Recent innovations in sheep breeding include the development of a flexible catheter at the National Center for Genetic Resource Preservation, Fort Collins, Colorado, that can be inserted through the ovine cervix, thus overcoming the barrier to effective AI in this species.

AI in sheep and goats is traditionally performed with fresh or cooled spermatozoa, with acceptable fertility results. However, use of foreign breeds, genetic improvement and the use of "safe" semen from other countries requires the use of frozen semen, to enable analyses for contaminants or diseases in the "donor" male to be completed before the semen doses are used for AI. Although the post-thaw motility of frozen semen from goats and sheep is usually considered acceptable, low fertility has been associated with its use in AI, mainly owing to a shortened lifespan of the spermatozoa.

\subsection{Intrauterine insemination in human fertility treatment}

It is estimated that $10-20 \%$ of couples wanting to conceive are unable to do so without some assistance. In $40 \%$ of cases, sub-fertility is due to female factors, with a further $40 \%$ being due to male factors. The remaining cases may be multifactorial or idiopathic in origin. The use of IUI is generally contraindicated in male factor infertility, with IVF or ICSI being the treatments of choice. Since spermatozoa must be able to reach the site of fertilization and the products of conception must be able to reach the uterus for implantation, female factor infertility due to blockage of the oviducts is better treated by IVF or ICSI than by IUI. The patient's own semen or donor semen may be utilized for these fertility treatments.

\section{Al - State of the art}

AI can help to improve reproductive efficiency in animals for food production or sport. We are living in a world of scarce resources where there is constant competition for water, food, land and energy. Since protein of animal origin continues to be one of the most important forms of nourishment for human beings, animals are an essential part of the ecosystem and must be husbanded in a sustainable fashion. Animal production not only "competes" with human beings for the aforementioned resources, but also produces large amounts of effluent and gaseous emissions which can affect the environment. Therefore, it is vital for the survival of the planet that all aspects of animal production are justified and optimized. Through grazing or browsing and the recycling of nutrients, animals also contribute to maintaining the landscape in a productive state.

The production of food of animal origin is based on breeding offspring to enter various husbandry systems. Therefore, one of the first points for optimization is in increasing reproductive efficiency, using an holistic approach. Females should be bred for the first time at an appropriate age to ensure the birth of healthy offspring and optimum lactation, without compromising the health of the female. Subsequent breeding attempts should also be timed appropriately to balance the metabolic requirements of lactation and early pregnancy. Females not conceiving or showing early embryonic loss should be identified at an early stage for re-breeding or culling. However, optimizing female reproduction demands a supply of spermatozoa. The spermatozoa must be readily available (i.e. can be 
stored), robust, and capable of fertilization, initiation of early embryonic development and regulation of placental formation, and there must be a means of delivery to an appropriate site in the female.

\section{Al in other species}

AI in non-domestic species presents several new challenges compared with domestic species. In many cases little is known about the reproductive biology of the species in question, and handling the animals may cause them stress, with the attendant risk of injury. The animals must be managed correctly for the establishment and maintenance of pregnancy. There are reports of successful AI in deer, buffalo and camelids.

\section{Future trends in Al}

It is highly probable that the use of AI in livestock will continue to increase. AI not only facilitates more effective and efficient livestock production, but can also be coupled to other developing biotechnologies, such as cryopreservation, selection of robust spermatozoa by single layer centrifugation, and sperm sex selection.

\subsection{Al in increasing the efficiency of livestock production}

Apart from some specialist sheep or goat units focussing on milk production for cheese and intensive meat production, farming of these species tends to be confined to marginal land that is unsuitable for crop production or grazing for dairy cattle. There has been limited selection for production traits. However, there is a resurgence of interest in them now in developed countries because of growing awareness that small ruminants could represent better utilization of scare resources than larger ones, such as cattle, while producing less methane and effluent. In many developing countries, sheep and goats are better suited to the climate than cattle, and it is culturally acceptable to eat their meat and milk products. Thus it is likely that there will be an upsurge in the use of AI in sheep and goats in the future, with an emphasis on improving production traits by the introduction of superior genes. However, it is essential that any A.I. scheme aimed at large scale improvement of the national herd must be supported by improved animal husbandry and animal health, otherwise the pregnancies resulting from AI will not go to term, and the offspring will either not survive or will fail to thrive. Many of the advanced ART are of little help in areas where basic husbandry skills are inadequate.

\subsection{Biomimetic sperm selection}

One potential disadvantage of $\mathrm{AI}$ is that the natural selection mechanisms within the female reproductive tract to select the best spermatozoa for fertilization may be bypassed when AI is utilized. Biomimetics is the use of technologies and/or processes that mimic a naturally occurring event. Several in vitro procedures have been suggested that could be used to mimic selection of good quality spermatozoa in the female reproductive tract and thus fit the definition of biomimetics in ART. These include sperm processing procedures such as swim-up, sperm migration, filtration and colloid centrifugation (reviewed by Morrell \& Rodriguez-Martinez, 2009). Of these methods, the one that is most applicable to livestock and human spermatozoa is colloid centrifugation. 


\subsubsection{Density gradient centrifugation}

Human spermatozoa for fertility treatment are usually processed to remove the seminal plasma and to select those of better quality. In most cases, this is achieved either by sperm migration, in which the more motile spermatozoa are separated from the rest of the ejaculate, or by density gradient centrifugation, where the most robust spermatozoa are selected. The benefits of density gradient centrifugation are as follows (Morrell, 2006):

i. Poorly motile and abnormal spermatozoa are removed,

ii. Sources of ROS (cell debris, leukocytes, epithelial cells and dead or dying spermatozoa) are removed;

iii. Sperm survival is improved during frozen and non-frozen storage;

iv. Bacterial contamination is controlled without antibiotics.

\subsubsection{Single layer centrifugation}

Density gradient centrifugation is seldom used when processing animal semen because of the limited volume of semen that can be processed at one time and the time taken to prepare the different layers. A novel sperm preparation technique, Single Layer Centrifugation (SLC) through a colloid, was developed at the Swedish University of Agricultural Sciences (SLU) to select the most robust spermatozoa from ejaculates. This method is similar to density gradient centrifugation (DGC), but is better suited for animal semen since it has been scaled-up to process whole ejaculates. The major applications for SLC-selection are similar to DGC end have been reviewed extensively by Morrell \& Rodriguez-Martinez (2010)

\subsection{Sex selection}

For many centuries, animal breeders and researchers have endeavoured to control the sex of the offspring born, for various reasons. Initially male offspring were preferred for meat production, because of the better feed conversion efficiency and lean-to-fat ratio of males, whereas females were preferred for dairy purposes, except that some males of high genetic merit were still required as sires. Couples may want a child of a specific sex to avoid the expression of sex-linked disorders.

Many methods have been proposed for separating X- and Y-chromosome bearing spermatozoa, based on physical properties, e.g. size of the sperm head, or functional properties e.g. swimming speed. However, the only method which has been shown to work reliably is that of selection and separation of spermatozoa whose DNA is stained with a bisbenzimidazole dye, H33342, using the sorting capacity of a flow cytometer (Morrell et al., 1988; Johnson et al., 1989). This method functions because the $X$ chromosome is larger than the $Y$, therefore taking up more of the DNA-specific stain and showing a higher fluorescence when the spermatozoa are passed through a laser beam. In bulls, for example, the difference in DNA content between the $\mathrm{X}$ and $\mathrm{Y}$ - chromosome is approximately $4.2 \%$. However, the process of sorting sufficient numbers for an insemination dose in the flow cytometer takes too long, since the stained spermatozoa must pass one at a time through a laser beam for detection of their DNA content. Moreover, the pregnancy rate after insemination of sexed bull spermatozoa is lower than with unsexed spermatozoa, making the procedure inefficient and expensive. Experience has shown that the staining profiles are highly individual, with the result that it is not possible to separate the $\mathrm{X}$ - and $\mathrm{Y}$-chromosome bearing spermatozoa efficiently from all males.

Alternative methods of sex selection are also being investigated. A company in Wales, Ovasort, has identified sex-specific proteins on the sperm surface and have raised antibodies 
to them. It is intended to use the antibodies to aggregate spermatozoa bearing a specific sex chromosome, thus enabling them to be removed from the general population.

A combination of ARTs would also be relevant for sperm sexing. Thus, the speed of flow sorting can be increased by first removing the dead and dying spermatozoa from the population, for example by density gradient centrifugation or single layer centrifugation. Such a combination may increase the "sortability" of sperm samples. Sufficient sexed spermatozoa may be obtained from flow sorting for IVF, thus generating embryos or blastocysts for subsequent transfer. However, methods of speeding up the selection process are needed if flow cytometry is to become useful for species other than the bovine.

\subsection{Sperm cryopreservation}

As previously mentioned, the ability of cryopreserved spermatozoa to retain their fertilizing ability varies widely between species. New cryoextenders and new protocols are being developed constantly in an effort to address this issue. One recent advance has been the introduction of dimethylsulphoxide and the amides formamide and dimethylformamide as cryoprotectants, in place of glycerol. These molecules seem to function better than glycerol for some individuals whose spermatozoa do not freeze well, for example, some stallions. One explanation for this observation is that these molecules are smaller than glycerol and therefore may cause less damage when they penetrate the sperm membrane. However, no method appears to be universally successful within one species. As far as turkey spermatozoa are concerned, it seems that the development of a successful freezing method will require more than new cryoprotectants and additives (Holt, 2000).

\subsection{Removal of viruses from ejaculates}

Viral infectivity can be removed from the semen of patients with viral infections such as HIV and hepatitis, by a sequential method of sperm preparation i.e. centrifugation on a density gradient followed by a "swim-up" (reviewed by Englert et al., 2004). Spermatozoa from virally infected men prepared by this method have been used in assisted reproduction attempts, apparently without sero-conversion of mothers or children. However, some studies with HIV report that density gradient centrifugation alone will not remove all viral infectivity (Politch et al., 2004). Since spermatozoa may function as vectors for viruses (Chan et al., 2004), further work is required to investigate how closely different viral particles are associated with the sperm membrane with putative carry-over during processing. The double method of processing has also been successful in removing equine arteritis virus from an infected stallion ejaculate in a preliminary study (Morrell \& Geraghty, 2006). SLC together with swim-up was used to reduce viral infectivity from boar semen spiked with porcine circo virus 2 (Blomqvist et al., 2011).

\subsection{Al in conservation biology}

It has been suggested that AI and other forms of ART could be useful for genetic conservation and preservation of rare breeds. Many of these technologies have been successful to some degree in a research setting, but none have produced results sufficient to effect population-wide improvements in genetic management (Morrow et al., 2009). Cryopreservation of semen has been the most widely applied ART in this respect, but much of the frozen semen in so-called gene banks has never been tested for fertility. A lack of suitable females or dearth of knowledge about the reproductive biology of the species 
involved may contribute to this deficit. However, long-term storage of frozen gametes of unknown fertility is not a sustainable policy for the conservation of rare breeds and endangered species. The development of in vitro methods of testing sperm fertility would contribute considerably to conservation efforts. Since the semen quality in these animals may be poor (Gamboa et al., 2009), techniques such as SLC of samples prior to AI could be of considerable benefit in conservation breeding.

\section{Conclusion}

AI revolutionized animal breeding in the 20th century, particularly in combination with sperm cryopreservation. The AI industry has developed dramatically in most domestic species in the last few decades and its use is now widespread in intensive animal production. The development of other associated technologies, such as sperm selection and sex selection, are predicted to create powerful tools for the future, both for domestic livestock breeding and for the purposes of conservation. AI will continue to play a role in fertility treatment for human patients, although it may be superseded by IVF or ICSI. It has been suggested that AI (in animals) is entering a new era where it will be used for the efficient application of current and new sperm technologies (Roca, 2006). Exciting possibilities are offered by emerging techniques, such as Single Layer Centrifugation, for improving sperm quality in AI doses as well as for increasing sperm survival during cryopreservation.

\section{References}

Bellinge, B.S., Copeland, C.M., Thomas, T.D., Mazzucchelli, R.E., O'Neil, G., Cohen M.J. (1986). The influence of patient insemination on the implantation rate in an in vitro fertilization and embryo transfer program. Fertil Steril,, Vol. 46, 2523-2526.

Chan, P.J., Su, BC., Kalugdan, T., Seraj, J.M., Tredway, D.R., King, A. (1994). Human papillomavirus gene sequences in washed human sperm deoxyribonucelic acid. Fertil Steril., Vol. 61, pp. 982-985.

Englert, Y., Lesage, B., van Vooren, J.P, Liesnard, C., Place, I., Vannin, A.S., Emiliani, S., Delbaere, A. (2004). Medically assisted reproduction in the presence of chronic viral disease. Hum. Reprod. Update, Vol. 10, pp. 149-162.

Gamboa, S., Machado-Faria M,, Ramalho-Santos J. (2009). Seminal traits, suitability for semen preservation and fertility in the native Portuguese horse breeds Puro Sangue Lusitano and Sorraia: Implications for stallion classification and assisted reproduction. Anim Reprod Sci.Vol.113, pp.102-113.

Gutsche, S., Wolff, M., von Strowitzki, T., Thaler CJ. (2003). Seminal plasma induces mRNA expression of IL-1 $\beta$, IL-6 and LIF in endometrial epithelial cells in vitro. Mol Hum Reprod, Vol. 9, pp. 785-791.

Holt, WV. (2000). Basic aspects of frozen storage of semen. Anim. Reprod. Sci., Vol. 62, 3-22.

Leboeuf, B., Delgadillo, JA., Manfredi, E., Piacere, A., Clement, V., Martin, P., Pellicer, M., Boue, P., de Cremoux, R. (2008) Management of Goat Reproduction and Insemination for Genetic Improvement in France Reprod Dom Anim, Vol. 43 (Suppl. 2), pp. 379-385 
Johnson, L.A., Flook, J.P. and Hawk, H.W. (1989). Sex preselection in rabbits: live births from $X$ and $Y$ sperm separated by DNA and cell sorting. Biol Reprod. Vol. 41, pp.199-203.

Morrell, J.M., Keeler, K.D., Noakes, D.E., MacKenzie, N.M. \& Dresser, D.W. (1988). Sexing of sperm by flow cytometry. Veterinary Record, Vol. 122, pp. 322-324.

Morrell, J.M. (2006). Update on semen technologies for animal breeding. Reproduction in Domestic Animals, Vol. 41, pp. 63-67.

Morrell, J.M. \& Geraghty, R.J. (2006). Effective removal of equine arteritis virus from stallion semen. Equine Veterinary Journal. Vol.38, pp. 224-229.

Morrell, J.M., Rodriguez-Martinez, H. (2009). Biomimetic techniques for improving sperm quality in animal breeding: a review. The Open Andrology Journal, Vol. 1, pp. 1-9.

Morrell, J.M., Rodriguez-Martinez, H. (2010). Practical applications of sperm selection techniques as a tool for improving reproductive efficiency. Veterinary Medicine International, doi:10.4061/2011/2984767

Morrow, C.J., Penfold, L.M,, Wolfe, B.A.(2009). Artificial insemination in deer and nondomestic bovids. Theriogenology. Vol. 71, pp. 149-65

Pellicer-Rubio, M., Combarnous, Y. (1998). Deterioration of goat spermatozoa in skimmed milk-based extenders as a result of oleic acid released by the bulbourethral lipase BUSgp60. J Reprod Fertil, Vol. 112, pp. 95-105.

Politch, J.A., Xu, C., Tucker, L., Anderson, D.J. (2004). Separation of human immunodeficiency virus type 1 from motile sperm by the double tube gradeint method versus other methods. Fertil. Steril., Vol. 81, pp. 440-447.

Robertson, S.A., Mayerhofer, G., Seamark, R.F. (1992). Uterine epithelial cells synthesize granulocyte-macrophage colony-stimulating factor and interleukin-6 in pregnant and nonpregnant mice. Biol Reprod, Vol. 46, pp. 1069-1079

Robertson, S.A., Sjoblom, C., Jasper, M.J., Norman, R.J., Seamark, R.F. (2001). Granulocytemacrophage colony-stimulating factor promotes glucose transport and blastomere viability in murine preimplantation embryos. Biol Reprod Vol. 64, pp. 1206-1215

Robertson, S.A. (2005). Seminal plasma and male factor signalling in the female reproductive tract. Cell Tissue Res, Vol. 322, pp. 43-52

Roca, J., Vazquez, J.M., Gil, M.A., Cuello, C., Parrilla, I., Martinez, E.A. (2006). Challenges in Pig Artificial Insemination. Reprod Dom Anim, Vol. 41 (Suppl. 2), pp. 43-53.

Salha, O., Sharma, V., Dada, T., Nugent, D., Rutherford, A.J., Tomlinson, A.J., Philips, S., Allgar, V., Walker, J.J. (1999). The influence of donated gametes on the incidence of hypertensive disorders of pregnancy. Hum Reprod, Vol. 14, pp. 2268-2273.

Tremellen, K.P., Valbuena, D., Landera, S. J., Ballesteros, A., Martinez, J., Mendoza, S., Norman, R.J., Robertson, S.A., Simon, C. (2000). The effect of intercourse on pregnancy rates during assisted human reproduction. Hum Reprod, Vol. 15, pp. 2653-2658.

Troedsson, M.H., Loset, K., Alghamdi, A.M., Dahms, B., Crabo, B.G. (2001). Interaction between equine semen and the endometrium: the inflammatory response to semen. Anim Reprod Sci, Vol. 68, pp. 273-278.

Wani, N.A., Billah, M., Skidmore, J.A. (2007). Studies on liquefaction and storage of ejaculated dromedary camel (Camelus dromedarius) semen. Anim Reprod Sci, Vol. 109, 309-318.

Wishart, G.J. (1985). Quantitation of the fertilising ability of fresh compared with frozen and thawed fowl spermatozoa. British Poultry Science, Vol. 26, pp. 375-380. 


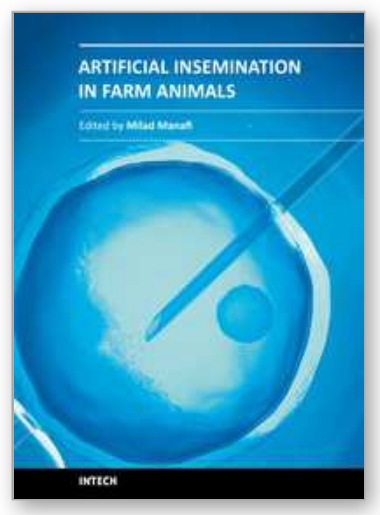

\author{
Artificial Insemination in Farm Animals \\ Edited by Dr. Milad Manafi
}

ISBN 978-953-307-312-5

Hard cover, 300 pages

Publisher InTech

Published online 21, June, 2011

Published in print edition June, 2011

Artificial insemination is used instead of natural mating for reproduction purposes and its chief priority is that the desirable characteristics of a bull or other male livestock animal can be passed on more quickly and to more progeny than if that animal is mated with females in a natural fashion. This book contains under one cover 16 chapters of concise, up-to-date information on artificial insemination in buffalos, ewes, pigs, swine, sheep, goats, pigs and dogs. Cryopreservation effect on sperm quality and fertility, new method and diagnostic test in semen analysis, management factors affecting fertility after cervical insemination, factors of noninfectious nature affecting the fertility, fatty acids effects on reproductive performance of ruminants, particularities of bovine artificial insemination, sperm preparation techniques and reproductive endocrinology diseases are described. This book will explain the advantages and disadvantages of using Al, the various methodologies used in different species, and how Al can be used to improve reproductive efficiency in farm animals.

\title{
How to reference
}

In order to correctly reference this scholarly work, feel free to copy and paste the following:

Jane M. Morrell (2011). Artificial Insemination: Current and Future Trends, Artificial Insemination in Farm Animals, Dr. Milad Manafi (Ed.), ISBN: 978-953-307-312-5, InTech, Available from:

http://www.intechopen.com/books/artificial-insemination-in-farm-animals/artificial-insemination-current-andfuture-trends

\section{INTECH}

open science | open minds

\section{InTech Europe}

University Campus STeP Ri

Slavka Krautzeka 83/A

51000 Rijeka, Croatia

Phone: +385 (51) 770447

Fax: +385 (51) 686166

www.intechopen.com

\section{InTech China}

Unit 405, Office Block, Hotel Equatorial Shanghai

No.65, Yan An Road (West), Shanghai, 200040, China

中国上海市延安西路65号上海国际贵都大饭店办公楼 405 单元

Phone: +86-21-62489820

Fax: $+86-21-62489821$ 
(C) 2011 The Author(s). Licensee IntechOpen. This chapter is distributed under the terms of the Creative Commons Attribution-NonCommercialShareAlike-3.0 License, which permits use, distribution and reproduction for non-commercial purposes, provided the original is properly cited and derivative works building on this content are distributed under the same license. 